\title{
The radiology of small bowel lesions
}

\author{
Oscar CRaig \\ F.R.C.S.I., D.M.R.D., F.F.R. \\ Consultant Radiologist, St Mary's Hospital, London, W.2; \\ and Bolingbroke Hospital, London, S.W.11
}

\begin{abstract}
Summary
The techniques of radiological examination of the small bowel are considered.

The importance of the routine barium meal and follow-through examination is stressed.

The vast majority of lesions present a diagnostic problem in interpretation rather than in detection.
\end{abstract}

Some examples of small bowel pathology are discussed and the need for interpretation in the light of the clinical picture is emphasized.

Important radiological signs to be appreciated are considered.

\section{Introduction}

Plain-film examination of the abdomen plays a very useful role in the investigation of small bowel lesions. Numerous contrast examinations are available to assist in the diagnosis of small bowel pathology:

(1) Barium meal and follow-through examination.

(2) Hypotonic duodenography.

(3) Small bowel enema.

(4) Barium enema with reflux into the terminal ileum.

(5) Angiography.

(6) Lymphangiography.

Vascular lesions may be demonstrated by angiography. In some cases of protein-losing enteropathy, lymphangiography may assist in the diagnosis of intestinal lymphangiectasis. These specialized procedures and the plain film changes will not be considered in this paper which will emphasize the radiological findings on barium examination.

\section{Hypotonic duodenography}

This was described by Mallet-Guy \& Jacquemet in 1963. Raia \& Kreel described their technique using the Scott-Harden tube in 1966. This doublelumen tube is passed into the stomach, and the inner tube manipulated into the second part of the duodenum. Four milligrams of oxyphenonium (Antrenyl) are given intravenously. Xylocain $(2 \mathrm{ml}$ of $4 \%$ solution diluted to $10 \mathrm{ml}$ ) is injected into the second part of the duodenum, after which barium is introduced. Three minutes after the intravenous injection of oxyphenonium the duodenal loop can be seen to become flaccid and immobile. This is assisted by the local application of xylocaine. A forced oxygen insufflation of the loop is then performed and spot films taken. It is hoped by this procedure to demonstrate minor distortions of the duodenal mucosa, lesions of the head of the pancreas, and papilla of Vater. Since the description of this technique, modifications have been made. Many authorities feel that it is adequate to produce duodenal relaxation with oxyphenonium and manipu late gastric air into the duodenum along with the barium taken conventionally during a barium meat examination. Supine spot films of the duodenal loo are then taken.

\section{Small bowel enema}

This was suggested by Schatzki in 1943. ScottHarden in 1960 described his technique using the double-lumen tube. Having passed the tube into the stomach and the inner tube as far as possible along the duodenal loop, $20-30 \mathrm{ml}$ of non-flocculating barium diluted with water to $60-90 \mathrm{ml}$ are injected. This is followed by $\frac{3}{4}-1$ pint of water, to which a small teaspoonful of magnesium sulphate has been added. The whole small bowel should be outlined within 10-15 min of the start of the injection. The barium passes to the caecum but leaves behind small bowel outlined by mucosal adhesion. There is thus a double contrast examination and the tendency for loops to obscure each other is reduced. This technique gives good results in the jejunum and proximal ileum, but frequently fails to give good visualization of the terminal ileum. To improve this, Trickey, Halls \& Hodgson (1963) described the use of a $0.75 \%$ solution of prepacol, as the 'flushing' agent, following the introduction of barium.

Whereas this technique may supplement the routine barium meal and follow-through, it has never been suggested that it should replace it. In the examination of patients suspected of having a small bowel lesion, it is only rarely that the difficulty lies 
in demonstrating the lesion. This may certainly be so, in some cases of small benign tumours, angioma and carcinoid tumours, but in the vast majority the difficulty lies in the correct interpretation of abnormal findings. These abnormal findings can usually be readily demonstrated by the routine barium meal and follow-through examination, and the conditions described in this paper have been examined by this method.

\section{Technique}

The patient takes the barium orally, and an examination of the oesophagus, stomach and duodenum are performed in the routine manner. Four to $8 \mathrm{oz}$ of barium are given according to the presenting problem. Films of the bowel can be taken routinely, at $2,4,6$ and $24 \mathrm{hr}$, but this timing can be modified. Earlier or more frequent films may be required. Maxolon $10 \mathrm{mg}$ orally $15-20 \mathrm{~min}$ before the examination produces 'intestinal hurry' and enables visualization of the small bowel to the terminal ileum within 1-2 hr. This can be of great advantage where the time factor is important to the radiologist. However, where disorders of motility are likely, no bowel stimulants should be given.

In cases where the malabsorption syndrome is likely then a non-flocculable barium, e.g. Raybar, should be used. Where a dissacharidase deficiency is possible, $20 \mathrm{~g}$ of lactose should be added to the barium mixture. Some authorities think that lactose should be added in all cases for its screening value in this respect.

Spot compression films of the terminal ileum should be taken as a large number of small bowel lesions involve this area. When doubt arises about the terminal ileum in the conventional followthrough examination, this area can also be visualized by a barium enema, efforts being made to produce reflux into the terminal ileum.

No attempt will be made to cover all the possible lesions of the small bowel, which is beyond the scope of this paper. Selected conditions will be considered, and although some are rare, they are amongst those that it is one's daily task to either search for or exclude in routine examinations.

\section{Duodenal carcinoma}

In routine barium meal examinations, the whole of the duodenal loop should be examined. It is possible for the fourth part of the loop to be hidden behind the stomach, and in these cases, to visualize this area it is necessary to take a film with the patient prone, lying on a compression pad. This will displace the stomach upwards, uncovering the fourth part of the duodenum and the duodenojejunal flexure. Carcinoma of the duodenum is said to be rare, but Jefferson (1916) remarked that inch for inch the duodenum is more likely to undergo cancerous change than the jejunum or ileum. Bockus (1964) states that carcinoma of the duodenum makes up $3 \%$ of all intestinal carcinomas. In a series of twenty-two malignant lesions of the duodenum, Serrano \& McPeak (1966) reported that a preoperative diagnosis was only made in nine. Hoffman \& Pack (1937) reported six cases of infra-ampullary duodenal neoplasms, none of which was diagnosed by experienced radiologists. Kreel \& Mackintosh reported four cases of duodenal carcinoma in Gut in 1968 and eight cases were recorded in the British Journal of Surgery (Craig, 1969). One of these cases was a 78-year-old man with a history suggestive of hiatus hernia. The patient complained of regurgitation of bilious fluid with lower retrosternal discomfort worse in bed and on stooping. There were no relevant physical findings but there was a weakly positive occult blood in the faeces. On examination, no hiatus hernia was seen, but there was gastrooesophageal reflux. Examination of the duodenal loop revealed a stricture in the descending limb, diagnosed as carcinoma, and confirmed at operation. A further case in a female aged 70 presented with a history of two attacks of vomiting but no abdominal pain. A neoplasm was found in the fourth part of the duodenum lying behind the stomach. In yet another case the lesion lay behind the stomach and was only found in the third barium examination. These cases illustrate the need for a high index of suspicion with regard to duodenal neoplasms on the part of the radiologist and his clinical colleagues and the necessity of a complete visualization of the duodenal loop in the routine barium examination.

\section{Duodenal ileus}

Obstruction of the duodenum due to compression by the superior mesenteric artery is considered by most physicians to be very rare. von Rokitansky first suggested this condition as a pathological entity in 1849. The following case was of interest as a diagnostic problem. The patient was a young woman of 23 who complained of epigastric fullness and vomiting after meals. No abnormality was found on clinical examination. There were no abnormal biochemical findings and the barium meal was negative. During the next year the vomiting increased and there was a loss of $2 \frac{1}{2}$ stones in weight. A repeat barium meal was performed during an attack and this showed an alteration in calibre in the third part of the duodenum with marked reversed peristalsis. There was a linear constriction where the superior mesenteric artery crossed the duodenum, not seen at the previous examination, and there was slight holdup of barium proximal to this area. The patient was examined prone and the barium passed satisfactorily 
through this area. The examination was repeated with bread soaked in barium and similar findings were reported.

At laparotomy, the superior mesenteric artery formed a tight band across the third part of the duodenum and the findings confirmed the radiological diagnosis of duodenal ileus.

Although this condition is rare in the experience of most clinicians, Bockus (1964) stated that he saw six cases of distal duodenal obstruction annually, of which $50 \%$ were due to vascular obstruction.

It is important for both radiologists and clinicians to appreciate that in this condition there is intermittent delay in the passage of duodenal contents and that when an initial barium examination is negative, the meal should be repeated during an attack.

\section{Disaccharidase deficiency}

Patients with this complaint present with vague abdominal pain, flatulence and diarrhoea. Lactase deficiency is the most common cause for this and deficiency of maltase and sucrase are very rare indeed. To test for hypolactasia $20 \mathrm{~g}$ of lactose are added to the barium mixture and the patient lies on the right side for $1 \mathrm{hr}$. An abdominal film is then taken with the patient supine. If there is a lactase deficiency, the following changes are seen:

(1) Intestinal hurry-the barium mixture will be well along the colon by $1 \mathrm{hr}$.

(2) The whole of the small bowel and part of the large bowel are filled with very diluted barium, as water is 'attracted' into the bowel lumen.

\section{Malabsorption syndrome (Fig. 1)}

The radiological changes are in the vast majority of cases non-specific and consist of flocculation of barium, a disordered mucosal pattern, and alterations in calibre of the small bowel. Raybar, a nonflocculable mixture, shows the mucosal pattern best and demonstrates thickened and coarsened folds in the jejunum. The rate of transit of barium through the bowel may be retarded. The best radiological sign of malabsorption is dilatation of small bowel coils. It was shown by Laws in 1964 that dilatation of jejunal coils is more closely related to the clinical severity of the malabsorption than any other radiological sign. Normal jejunum has a diameter of up to $25 \mathrm{~mm}$ and anything over $30 \mathrm{~mm}$ in the adult can be considered as pathologically dilated.

The list of causes of the malabsorption syndrome is long, and apart from idiopathic steatorrhoea, includes Crohn's disease, scleroderma, Whipple's disease, Hodgkin's disease and lymphosarcoma. Pancreatic, biliary and post-operative causes, e.g.

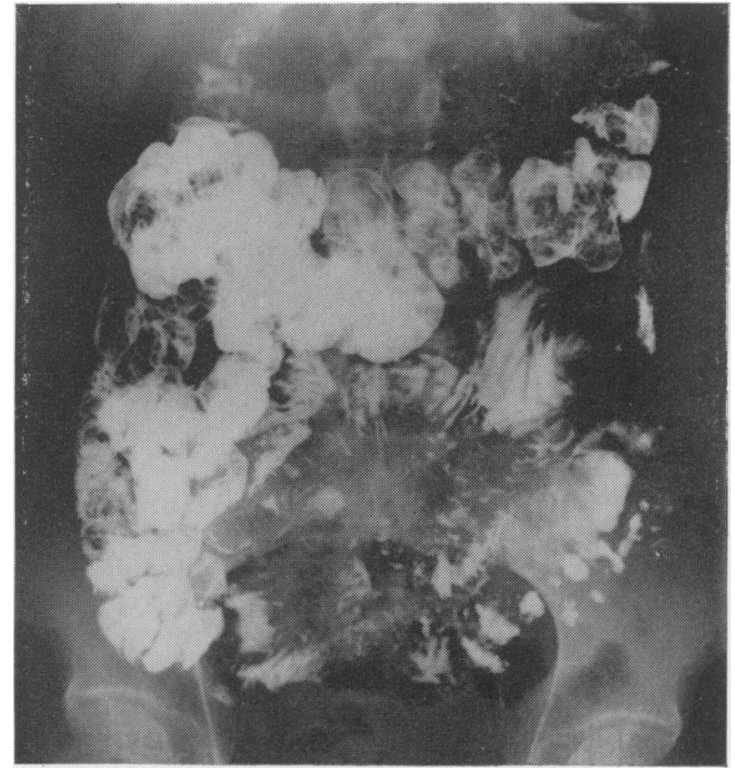

FIG. 1. Alteration in bowel calibre, mucosal thickening, flocculation and segmentation in a case of idiopathic steatorrhoea.

blind loops, resections, etc., also need consideration.

It is the duty of the radiologist to have these in mind during his examination and search for any signs that might indicate the underlying aetiology. Some of the cases to be quoted in this paper may illustrate this point.

\section{Regional enteritis}

The terminal ileum is the most frequent site of involvement in regional enteritis and this area is easily visualized by spot films in a routine barium meal and follow-through examination. Early cases of involvement can be difficult to detect as there may be only a minimal alteration in the mucosal pattern or peristaltic activity. Later signs are thickening of the mucosa, patchy filling defects and narrowing of the lumen. The string-sign is almost pathognomonic of regional enteritis, but does not always indicate a non-reversible phase of the disease. It can also be seen due to spasm and irritability. Although complete intestinal obstruction is rare, fistulae and perforation may occur.

Fig. 2 shows the findings in a 10 -year-old patient who had Crohn's disease involving a large area of jejunum and also the duodenum.

Approximately forty-seven cases of Crohn's disease of the duodenum have been described in the literature, but it seems highly likely that this is not a true estimate of its frequency owing to the reluctance of many authors to report single cases. In a review of 


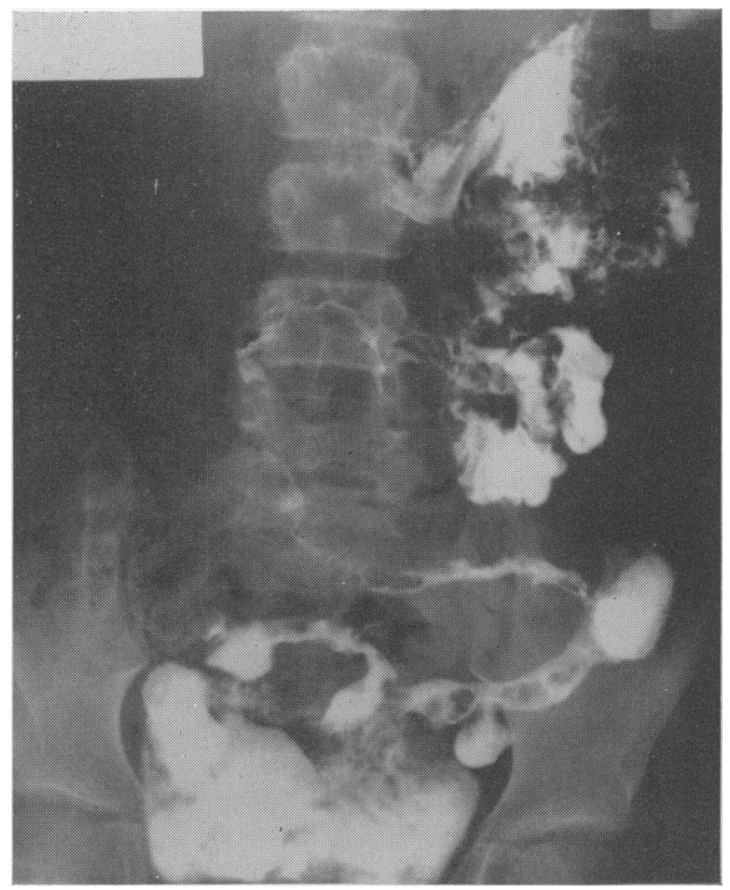

FIG. 2. Stricture formation, mucosal irregularity with 'cobble stone' deformity in regional enteritis. Note the 'pseudo-diverticula'. These are normal areas of bowel between the 'strip areas'.

148 cases of small bowel Crohn's disease by Marshak (1961), four cases had duodenal involvement. Thirty-one cases of duodenal Crohn's disease were reviewed by Pryce-Davies in the Journal of Clinical Pathology in 1964. Of these, only three were confined to the duodenum, the remainder having lesions elsewhere in the small bowel. A routine barium meal and follow-through wiil usually demonstrate Crohn's disease quite readily at the time the patient presents with symptoms. If there is any doubt about the terminal ileum or caecum, a barium enema can be performed to show both these areas.

\section{Lymphosarcoma and Hodgkin's disease}

Fig. 3 illustrates the case of a 48-year-old female patient with Hodgkin's disease. In 1963 this patient presented with severe cramping abdominal pain, nausea, vomiting and diarrhoea. A jejunal ulcer was found at laparotomy and excised. The histology was that of non-specific ulceration. One year later the symptoms recurred. The barium meal and follow through showed a jejunal ulcer just beyond the duodeno-jejunal flexure and irregularity of small bowel loops below this. At laparotomy four ulcers were found in the small bowel. The histology demonstrated malignant Hodgkin's disease.

Fig. 4 shows the contrast examination on a 70 -

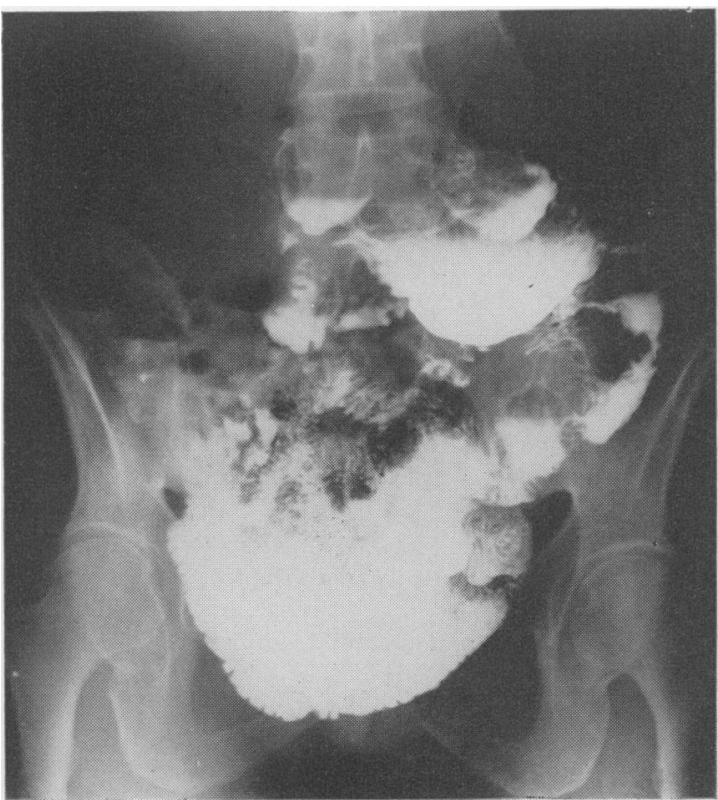

FIG. 3. Areas of ulceration and stricture formation high in the jejunum due to Hodgkin's disease.

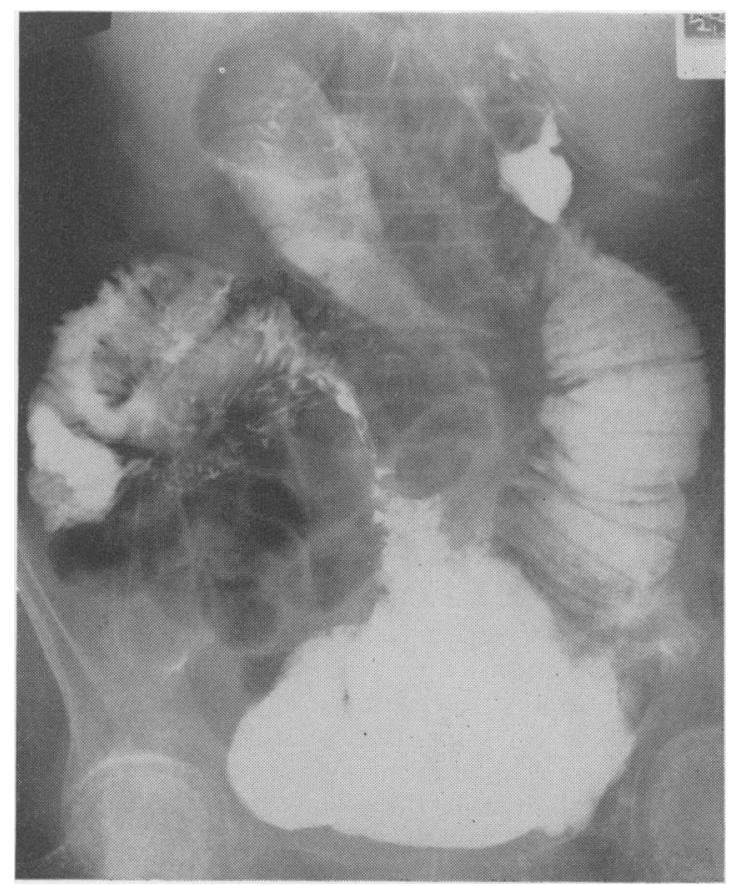

FIG. 4. Areas of narrowing and marked dilatation seen in lymphosarcoma. 
year-old female patient on whom a clinical diagnosis of idiopathic steatorrhoea had been made. The films show an abnormal jejunal pattern with areas of narrowing and dilatation of the bowel. There was no response to a gluten-free diet. The patient's condition deteriorated and she developed attacks of abdominal distention with vomiting and an increase in the diarrhoea. There was a poor response to steroid therapy and laparotomy was undertaken. Four strictures were found in the small bowel. The histology report was lymphosarcoma.

Malignant intestinal lymphoid tumours occur most frequently in the stomach. Dawson, Cornes \& Morson (1961) reported thirty-seven cases of small bowel lymphomata seen in three major centres over the past 25 years. They reviewed 140 cases in the literature. These cases were divided into:

Giant follicle lymphoma.

Lymphosarcoma.

Hodgkin's disease.

Hodgkin's sarcoma.

Reticulum cell sarcoma.

The Mayo Clinic reported 659 small bowel tumours of which $8 \%$ were malignant lymphomata (Good, 1963). Three varieties of lymphomatous involvement have been described:

(1) Mucosal type-like an adenocarcinoma.

(2) Aneurysmal type.

(3) Diffuse type.

Lymphomatous involvement of the small intestine can closely mimic adult coeliac disease both radiologically and clinically. There may be steatorrhoea and laboratory evidence of malabsorption may be identical in both. Even biopsy with a Crosby capsule may show flattened mucosa in both. However, failure to respond to a gluten-free diet should always suggest a diagnosis other than primary idiopathic steatorrhoea. The major clinical presentations of small bowel lymphomata consist of:

$\begin{array}{ll}\text { Intestinal obstruction } & 67 \% \\ \text { Intestinal bleeding } & 53 \% \\ \text { Abdominal mass } & 31 \% \\ \text { Perforation } & 11 \%\end{array}$

Clinically, the triad of a short clinical history, a palpable tumour and the absence of significant wasting should suggest a lymphoid tumour rather than a carcinoma.

Radiologically, the presence of multiple strictures will exclude an idiopathic steatorrhoea. Areas of quite marked dilatation may occur in lymphosarcoma due either to direct infiltration of the bowel or involvement of local nerve plexuses.

\section{Whipple's disease}

This was first described in 1907 at the Johns
Hopkins Hospital following the post mortem on a 36-year-old physician missionary (Whipple, 1907). In 1957 Ammann collected seventy-two acceptable cases from the world literature, all but twelve reported since 1942. Since 1947 there have been at least forty more reported cases. The symptoms appear in the middle or later years of life and there is a male preponderance of 8: 1 (Bockus, 1964).

On barium meal examination, the appearances may strongly resemble those of idiopathic steatorrhoea, but usually the segmentation and flocculation are not so marked. However, some important signs that may suggest this condition are:

(1) Arthritis, which frequently antedates the gastro-intestinal symptoms for some years. This affects both large and small joints. The arthritis may decrease in severity with the onset of gastro-intestinal disturbances.

Radiologically, involvement of the sacroiliac joints may be seen. These may show partial fusion or irregularity, narrowing and sclerosis.

(2) Mediastinal glandular enlargement may occur.

(3) Bowel loops may be displaced by enlarged abdominal glands. There may be an extreme degree of wasting and weakness. Pigmentation of the skin is frequently seen. Enlarged cervical, axillary or groin glands may be found

\section{Henoch-Schönlein purpura}

Fig. 5 shows the barium findings in a 17 -year-old boy who presented with a purpuric rash. While in the hospital he developed severe abdominal pain, diarrhoea and melaena. The barium examination showed mucosal irregularity with oedema, involving the duodenum, jejunum and ileum. 'Thumbprinting' and 'picket fence deformity' are radiological signs of intramural haemorrhage. Haemorrhage into the wall may precipitate an intussusception leading to intestinal obstruction.

\section{Carcinoid tumour}

Fig. 6 shows multiple filling defects involving the small bowel due to 'masses' encroaching on the bowel lumen from the bowel wall. These were due to carcinoid tumour deposits. The patient complained of diarrhoea, flushing attacks and palpitations. In this case, these symptoms were more marked during defaecation, as the result of multiple deposits in the rectal wall.

Carcinoid tumours were first described by Merling in 1808. The majority are found in the appendix or terminal ileum. Of 659 tumours of the small bowel reviewed between 1938 and 1957 at the Mayo Ciinic, $23 \%$ were carcinoids (Good, 1963). The tumours may be single or multiple, benign or malignant. 


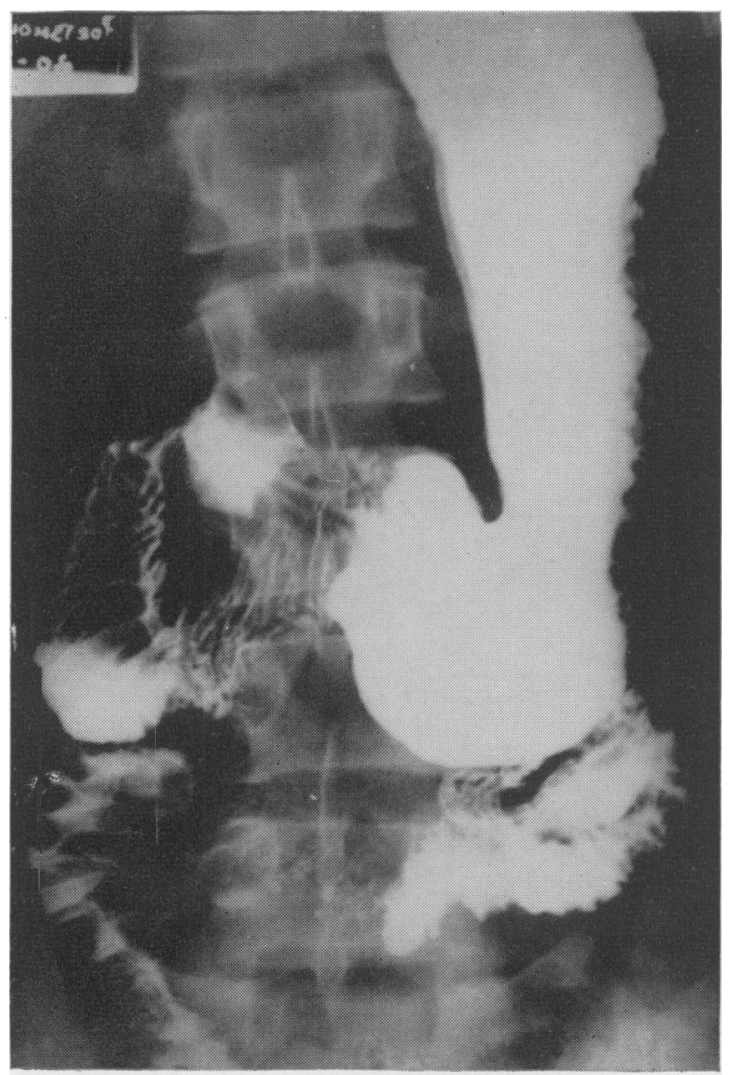

FIG. 5. 'Picket fence' deformity in the jejunum and altered duodenal outline due to intra-mural haemorrhage in Henoch-Schönlein purpura.

Although they make up less than $1 \%$ of the tumours of the total gastro-intestinal tract, they form $20 \%$ of all the malignant tumours of the small bowel. They may produce symptoms due to:

(1) Mechanical effects.

(2) Metastases.

(3) Pharmacological effects. It is said that $28 \%$ of malignant carcinoids secrete serotonin.

Good in 1963 stated that attempts at radiological diagnosis of carcinoid tumours had been very unsuccessful at the Mayo Clinic. The difficulty arises in that the tumour is often very small and that signs are only produced when the tumour extends into the neighbouring glands, causing kinking of the bowel, abdominal masses or obstruction. A moderate fibrotic reaction occurs within and about the tumour. Kinking of the bowel, although not specific, is certainly highly suggestive of carcinoid tumour.

\section{Endometriosis}

This most commonly affects the rectum or sigmoid colon, but it may also affect the small bowel. The history of abdominal pain, nausea, diarrhoea and sometimes abdominal distention, with exacerbations at menstruation suggests the diagnosis. The radiological changes are usually non-specific but barium examination may show bowel irregularity due to single or multiple masses encroaching on and distorting the bowel lumen.

\section{Scleroderma}

Involvement of the small bowel may occur in scleroderma and most often accompanies oesophageal involvement. However, on rare occasions, small bowel lesions may occur without obvious radiological change in the oesophagus. For this reason it is worthwhile performing a barium meal and follow-through even in cases where the oesophagus appears normal radiologically.

Fig. 7 shows the findings in a 50-year-old patient who presented with dysphagia but who also had steatorrhoea. Radiologically, there may be dilation of bowel loops, coarsening and thickening of intestinal mucosa. Areas of fibrous narrowing may also occur. There is evidence of bowel atony, with delay in the passage of barium through the small bowel, and often delay in gastric emptying. Pseudodiverticula have been described in the large bowel.

The general systemic manifestations, with skin involvement, lung changes and often Raynaud's type lesions suggest the diagnosis.

\section{Meckel's diverticulum}

Although frequently searched for, it is not common to demonstrate a Meckel's diverticulum by barium meal and follow-through examination. This is partly due to the overlapping of bowel loops, but mostly due to the fact that the diverticulum has a wide mouth and does not retain barium in its lumen.

Fig. 8, however, shows the findings in a patient with recurrent abdominal pain and distention, diarrhoea and positive occult blood. A Meckel's diverticulum was found on a long stalk lying between two coils of jejunum. Irregularities at the base of the diverticulum were due to associated involvement by Crohn's disease.

\section{Eosinophilic enteritis}

The first radiological description of this condition was by Herrera \& de la Guardia in 1948, although the first reference to the condition was made by Mock in 1931. The condition may present as a localized polypoid tumour, most often involving the pyloric antrum, or as a diffuse eosinophilic infiltration involving scattered areas of the stomach or small bowel. A blood eosinophilia is most often seen with this latter variety and an allergic history can often be elicited. 


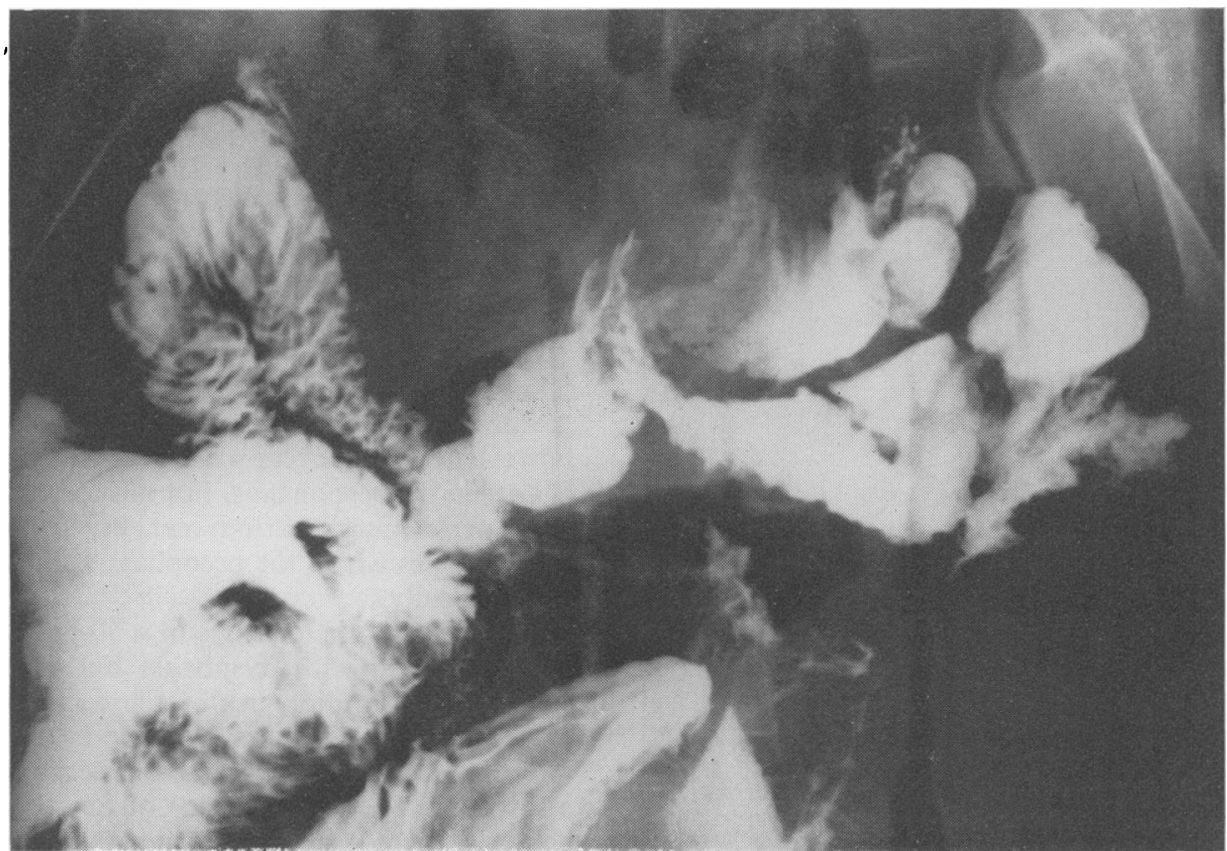

FIG. 6. Irregularity of bowel, kinking and stricture formation due to carcinoid tumour.

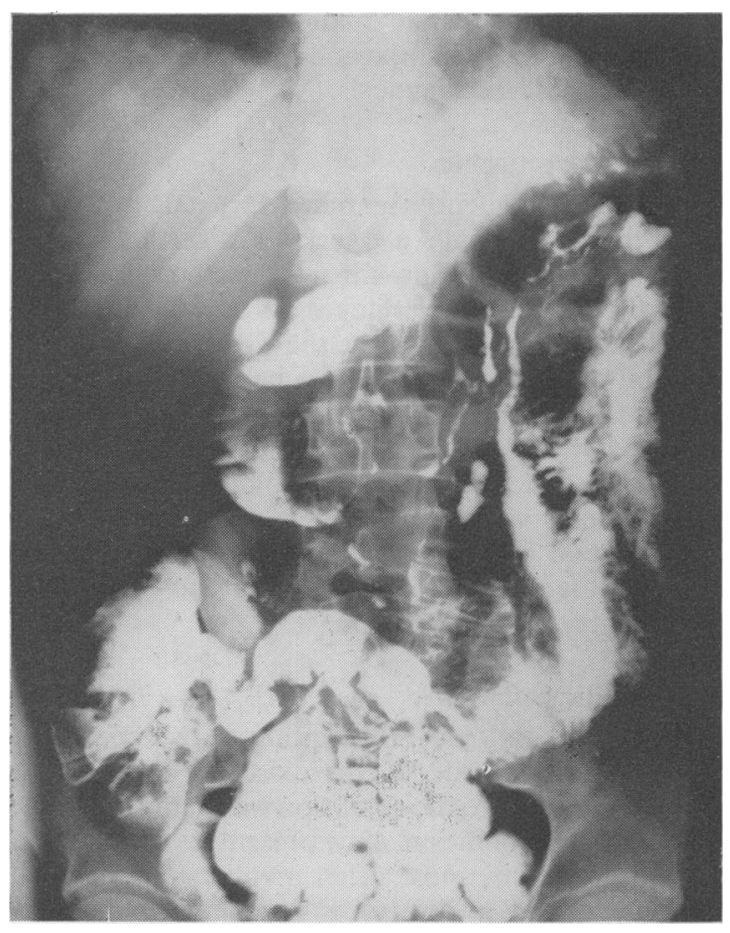

Fig. 7. Areas of fibrous narrowing and mucosal irregularity in scleroderma.

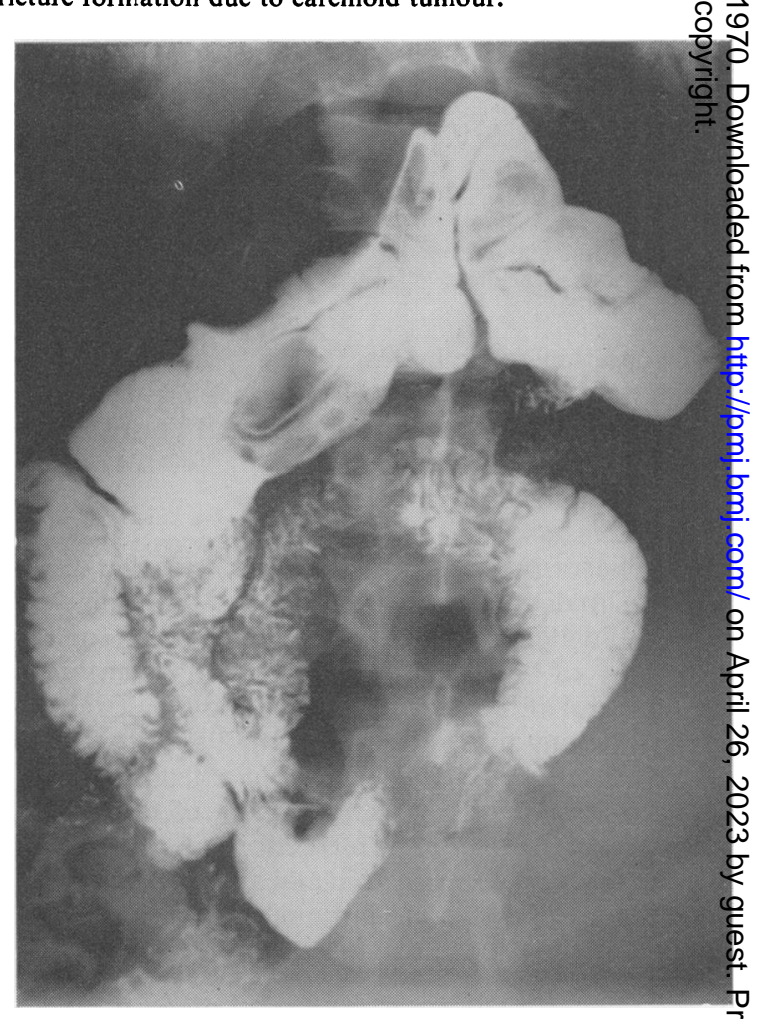

FIG. 8. A Meckel's diverticulum on a long stalk and lying between two coils of jejunum. Filling defects in the surrounding bowel were due to associated Crohn's disease. 
Radiologically, there may be a filling defect on barium examination or irregularity, oedema and narrowing of scattered areas of small bowel. These changes may resemble regional enteritis, lymphosarcoma or Hodgkin's disease. The diagnosis is suggested if these radiological changes are found in a patient with a history of recurrent abdominal pain, vomiting, diarrhoea and often an allergic history and a blood eosinophilia.

\section{Conclusion}

A number of lesions involving the small bowel have been considered. The cases described illustrate that the main difficulty is not in demonstrating the lesion but in interpreting the abnormal findings. It is especially important in these cases that the radiological changes are considered in conjunction with the clinical history and physical findings. Certain helpful radiological points should, however, be emphasized:

(1) The importance of constantly viewing the complete duodenal loop in a routine barium examination.

(2) The consideration of vascular duodenal compression in otherwise negative cases. The evaluation of 'cog-wheel' duodenal movement and the necessity of examining patients during an attack.

(3) The careful examination of the duodenum in high jejunal Crohn's disease.

(4) The importance of small bowel 'kinking' due to fibrosis in the search for carcinoid tumours.

(5) The value of a barium examination of the small bowel in cases of scleroderma, even in the presence of a normal oesophagus.

(6) In any malabsorption pattern, the presence of strictures, ulcers, filling defects or areas of aneurysmal dilatation will exclude a simple primary idiopathic steatorrhoea and suggest either a malignant or lymphomatous small bowel pathology. The presence of glandular enlargement and involvement of sacro-iliac joints may suggest Whipple's disease.

(7) Clinically, failure of a malabsorption syndrome to respond to a gluten-free diet should raise the suspicion of a lesion other than idiopathic steatorrhoea.

\section{Acknowledgments}

I am grateful to the many colleagues who referred cases for investigation and also express my thanks to Dr P. Cardew, Photographic Department of St Mary's Hospital Medical School for his help.

\section{References}

Amman, R.W. (1957) Zur Differentialdiagnose, Pathogenese und Atiologie des Morbus Whipple. Helvetica medica acta, 24, 118.

Bockus, H.L. (1964) Gastroenterology, 2nd edn, Vol. 2, p. 188. Saunders, Philadelphia.

Craig, O. (1969) Duodenal carcinoma. British Journal of Surgery, 56, 39.

Dawson, I.M.P., Cornes, J.S. \& Morson, B.C. (1961) Primary malignant lymphoid tumours of the intestinal tract. British Journal of Surgery, 49, 80.

Good, C.A. (1963) Tumors of the small intestine. American Journal of Roentgenology, Radium Therapy, and Nuclear Medicine, 89, 685.

Herrera, J.M. \& De la Guardia, J. (1948) Un raro caso de oesinofilia gastro-intestinal motivadora de un cuadro organico de estenosis pilorica. Archivos de Hospital de Santo Tomás, 3, 19.

Hoffman, W.J. \& Pack, G.T. (1937) Cancer of the duodenum: clinical and roentgenographic study of 18 cases. Archives of Surgery, 35, 11.

JefFerson, G. (1916) Carcinoma of the suprapapillary duodenum causally associated with pre-existing simple ulcer. British Journal of Surgery, 4, 209.

Kreel, L. \& Mackintosh, C. (1968) Carcinoma of the duodenum. Gut, 9, 222.

LAws, J.W. (1964) The small intestine. Recent Advances in Radiology (Ed. by T. Lodge), 4th edn, p. 108. Churchill, London.

Mallet-Guy, P. \& Jacquemet, P. (1963) Résultats de 500 cas de duodénographie hypotonique. Journal de radiologie, d'électrologie et de médicine nucléaire, 44, 249.

MaRSHAK, R.H. (1961) Roentgen findings in lesions of the small bowel. American Journal of Digestive Diseases, 6, 1084.

Merling (1808) Quoted by Steger, C. \& Avanzini, C. (1953) Ricerche isotopatologiche ed istochimiche sui carcinoidi. Rivista di anatomia patalogica e di oncologia, 7, 1091

Mock, H.E. (1931) Infective granuloma: non-specific chronic tumour-like productive inflammations of the gastrointestinal tract. Surgery, Gynecology, and Obstetrics with International Archives of Surgery, 52, 672.

Pryse-Davies, J. (1964) Gas-distension, double-contrast duodenography using the Scott-Harden gastroduodenal tube. Gut, 7, 420.

Rala, S. \& KReel, L. (1966) A Manual of Pathological Anatomy, Vol. 2. Sydenham Society, London.

Serrano, J.F. \& McPeak, C.J. (1966) Primary neoplasms of the duodenum. Surgery, 59, 199.

Schatzki, R. (1943) Small intestinal enema. American Journal of Roentgenology, Radium Therapy, and Nuclear Medicine, 50, 743.

SCOTT-HARDEN, W.G. (1960) Examination of the small bowel. Modern Trends in Diagnostic Radiology (Ed. by J. W. McLaren), 3rd series, p. 84. Butterworths, London.

Trickey, S.E., Halls, J. \& Hodson, C.J. (1963) A further development of the small bowel enema. Proceedings of the Royal Society of Medicine, 56, 1070.

Whipple, G.H. (1907) A hitherto undescribed disease characterized anatomically by deposits of fat and fatty acids in the intestinal and mesenteric lymphatic tissues. Bulletin of the Johns Hopkins Hospital, 18, 382. 